\title{
Manual therapies for cervicogenic headache: a systematic review
}

\author{
Aleksander Chaibi • Michael Bjørn Russell
}

Received: 21 December 2011/ Accepted: 7 March 2012/Published online: 30 March 2012

(C) The Author(s) 2012. This article is published with open access at Springerlink.com

\begin{abstract}
This paper systematically reviewed randomized clinical trials (RCT) assessing the efficacy of manual therapies for cervicogenic headache (CEH). A total of seven RCTs were identified, i.e. one study applied physiotherapy \pm temporomadibular mobilization techniques and six studies applied cervical spinal manipulative therapy (SMT). The RCTs suggest that physiotherapy and SMT might be an effective treatment in the management of $\mathrm{CEH}$, but the results are difficult to evaluate, since only one study included a control group that did not receive treatment. Furthermore, the RCTs mostly included participant with infrequent CEH. Future challenges regarding CEH are substantial both from a diagnostic and management point of view.
\end{abstract}

Keywords Randomized clinical trials - Manual therapies . Physiotherapy · Chiropractic · Cervicogenic headache ·

Treatment

\section{Introduction}

Cervicogenic headache (CEH) is a secondary headache characterized by unilateral headache and symptoms and signs of neck involvement [1-5]. It is often worsened by neck movement, sustained awkward head position or

A. Chaibi $(\bowtie) \cdot$ M. B. Russell

Head and Neck Research Group, Research Centre,

Akershus University Hospital, 1478 Lørenskog, Oslo, Norway

e-mail: aleksander.chaibi@medisin.uio.no

M. B. Russell

Institute of Clinical Medicine, Akershus University Hospital,

University of Oslo, 1474 Nordbyhagen, Norway external pressure over the upper cervical or occipital region on the symptomatic side $[1,3]$.

The prevalence of CEH varies in the general population depending on the diagnostic criteria, i.e. $1.0 \%$ applying six positive criteria of the Cervicogenic Headache International Study Group (CHISG) and $4.6 \%$ when only five criteria were used, while it was $2.5 \%$ applying the International Headache Society (IHS) criteria [3, 5-8]. A recent epidemiological survey found that the prevalence was $0.13 \%$ in men and $0.21 \%$ in women applying three or more major CHISG criteria $[9,10]$. Thus, along with different diagnostic criteria, it is likely that other methodological differences play a role.

The pathogenesis of CEH may originate from various anatomic structures in the cervical spine. Convergence of afferents of the trigeminal and upper three cervical spinal nerves onto the second-order neurons in the trigeminocervical nucleus in the upper cervical spinal cord is likely to lead to the headache $[11,12]$. The craniovertebral junction is stabilized by joint capsules, tectorial membrane, transverse and alar ligaments [13]. A proton-weighted magnetic resonance imaging (MRI) study of people with $\mathrm{CEH}$, whiplash-associated headache or migraine was analyzed blinded and identified no significant differences in the three groups [14]. Furthermore, the site of the CEH was not correlated with the site of signal intensity changes of the alar and transverse ligaments. One study suggests that lower cervical disc prolapse may cause CEH [15], but the results could not be confirmed as no specific MRI changes of cervical discs or craniovertebral ligaments were found in CEH [14]. Muscle tenderness is likely to play a role and is more pronounced on the pain than the non-pain site, i.e. pericranial tenderness was recorded according to the tenderness score of eight pairs of pericranial muscles and tendon insertion points, each scored on a four-point scale 
(0-3) at each location, and the tenderness score on the pain and non-pain sides was compared [10]. However, so far the pathogenesis and etiology of $\mathrm{CEH}$ remain a challenge.

Due to insufficient pharmacological treatment strategies, medication overuse is frequent and likely secondary to the pain rather than a confounding factor, as the medication overuse is of shorter duration than the duration of the $\mathrm{CEH}$ [10]. A 3-year follow-up of people with $\mathrm{CEH}$ from the general population found no improvement [16], while people from the general population with headache attributed to chronic rhinosinusitis or medication overuse headache improved after a short advice [16, 17].

Thus, due to muscle tenderness and possibly not yet identified local factor in the cervical spine, it might be that manual therapies can alleviate $\mathrm{CEH}$, along with blockage of the greater occipital nerve (GON), which is the only effective pharmacological treatment so far [18, 19]. This paper systematically review randomized clinical trials (RCT) assessing the efficacy of manual therapies for $\mathrm{CEH}$.

\section{Methods}

The literature search was done on CINHAL, Cochrane, Medline, Ovid and PubMed. Search words were cervicogenic headache and chiropractic, manipulative therapy, massage therapy, osteopathic treatment, physiotherapy or spinal mobilization. All RCT written in English using either of the manual therapies on $\mathrm{CEH}$ were evaluated. $\mathrm{CEH}$ was preferentially classified according to the criteria of the IHS from 1988 or its revision from 2004, or according to the Cervicogenic Headache International Study Group (CHISG) [1-5]. Table 1 shows the diagnostic criteria for CEH. The studies had to evaluate at least one $\mathrm{CEH}$ outcome measure, i.e. pain intensity, frequency, or duration. The methodological quality of the included RCT studies was assessed by the first author. Table 2 shows that the evaluation covers study population, intervention, measurement of effect, data presentation and analysis and the maximum score is 100 points, and $\geq 50$ points is considered to be methodology of good quality [20-23].

\section{Results}

The literature search identified seven RCT on CEH that met our inclusion criteria. One study applied physiotherapy \pm temporomadibular mobilization techniques and six studies applied cervical spinal manipulative therapy (SMT) [24-30]. Four studies were conducted by chiropractors, two studies by physiotherapists and one by a physician. RCTs studies on massage therapy, spinal mobilization or osteopathic intervention on $\mathrm{CEH}$ were not identified.
Methodological quality of the RCTs

Table 3 shows the methodological score of the included RCT studies. The score varied from 50 to 81 points.

Randomized controlled trials (RCT)

Table 4 shows details from the seven RCT studies regarding study population, intervention and results in relation to headache frequency, duration and intensity, while other results are presented in the text.

\section{Physiotherapy}

The Dutch study was conducted by experienced physiotherapists with unblinded treatment and outcome measures [30]. The participants were diagnosed with $\mathrm{CEH}$ by a neurologist according to the criteria of the International Headache Society (IHS) [5]. Participants were excluded, if ever received temporomandibular disorder (TMD)/orthodontic treatment or experienced neuropathic head pain. The primary end point was headache intensity while TMD complaints (mouth opening, pain and range, deviation, sounds and pain threshold of anterior temporal muscles) and neck disability were secondary end points. Both TMD complaints and neck disability improved statistically significantly in the experimental group as compared to conventional physiotherapy group at 3- and 6-month follow-up ( $p<0.001$ in both comparisons).

\section{Cervical spinal manipulative therapy}

The Danish study was conducted by a chiropractor with unblinded treatment and blinded analysis of outcome measures [24]. The participants were diagnosed by a physician according to the criteria of the IHS excluding the radiographic criterion [1]. Participants whom had not previously received SMT or had conditions contraindicated to SMT were excluded from the study. The primary endpoints were change in headache intensity, headache duration and NSAIDs consumption from pre-treatment at 2 weeks to post-treatment at week 6 . The consumption of NSAIDs was significantly reduced from pre-treatment to post-treatment in the cervical SMT group $(p<0.0005)$, but not in the soft tissue (ST) group, however, the reduction in consumption of NSAIDs was not statistically significantly different in the cervical SMT and ST groups $(p=0.14)$.

The 2nd Danish study was based on an extended study population from the 1 st Danish study [24, 25]. The methodology and end-points were similar, except that the pre-treatment period was reduced from 2 to 1 week and the statistical calculation was based on median rather than mean 
Table 1 Diagnostic criteria of cervicogenic headache

Cervicogenic Headache International Study Group [3]

Major criteria $^{\mathrm{a}}$

1. Symptoms and signs of neck involvement

a. Precipitation of head pain, similar to the usually occurring one:

i. By neck movement and/or sustained awkward head positioning, and/or:

ii. By external pressure over the upper cervical or occipital region on the symptomatic side

b. Restriction of range of motion (ROM) in the neck

c. Ipsilateral neck, shoulder, or arm pain of a rather vague nonradicular nature or, occasionally, arm pain of a radicular nature.

2. Confirmatory evidence by diagnostic anesthetic blockade

3. Unilaterality of the head pain, without side shift

Head pain characteristics

Ofher characteristics of some importance

Other features of lesser importance
4. a. Moderate-severe, non-throbbing, and nonlancinating pain, usually starting in the neck.

b. Episodes of varying duration

c. Fluctuating, continuous pain

5. a. Only marginal effect or lack of effect of indomethacin

b. Only marginal effect or lack of effect of ergotamine and sumatriptan

c. Female sex

d. Not infrequent occurrence of head or indirect neck trauma by history, usually of more than only medium trauma

6. a. Nausea

b. Phonophobia and photophobia

c. Dizziness

d. Ipsilateral "blurred vision"

e. Difficulties swallowing

f. Ipsilateral edema, mostly in the periocular area

International Classification of Headache Disorders-II [5]

A. Pain, referred from a source in the neck and perceived in one or more regions of the head and/or face, fulfilling criteria $\mathrm{C}$ and $\mathrm{D}$

B. Clinical, laboratory and/or imaging evidence of a disorder or lesion within the cervical spine or soft tissues of the neck known to be, or generally accepted as, a valid cause of headache

C. Evidence that the pain can be attributed to the neck disorder or lesion based on at least one of the following:

i. Demonstration of clinical signs that implicate a source of pain in the neck

ii. Abolition of headache following diagnostic blockade of a cervical structure or its nerve supply using placebo- or other adequate controls

D. Pain resolves within 3 months after successful treatment of the causative disorder or lesion

${ }^{\mathrm{a}}$ It is obligatory that one or more of phenomena $1 \mathrm{a}-1 \mathrm{c}$ are present

change. The consumption of NSAIDs was significantly reduced from pre-treatment to post-treatment in the cervical SMT group, but not in the ST group, however, the median reduction in consumption of NSAIDs was not statistically significantly different in the cervical SMT and ST groups $(p=0.14)$.

The Australian multicenter study was conducted by 25 experienced physiotherapists with unblinded treatment and blinded outcome measures [26]. The study participants were diagnosed according to the diagnostic criteria of CHISG by GPs or physiotherapists [4]. Those with bilateral headache, conditions that contraindicated to spinal manipulative treatment (SMT) or whom had received physiotherapy or chiropractic treatment for headache within the previous year were excluded. The primary endpoint was a change in headache frequency from baseline to immediately after treatment and 12 months after the intervention, while headache intensity, duration and neck pain were secondary end-points. Neck pain was reduced immediate post-treatment in the all intervention groups $(p<0.001-0.01)$, but was only maintained at 12-month follow-up in the exercise group and combined SMT and exercise group $(p<0.001-0.01)$. The median medication intake comparing baseline with 12-month follow-up was reduced $93 \%$ in the combined SMT and exercise group, $100 \%$ in the SMT and exercise groups, while it increased $33 \%$ in the control ( $p<0.015$ for all). The authors suggest that the treatment effect may be underestimated since $46 \%$ of controls received active intervention (unspecified) for their headache within the trial period.

An American study conducted by three experienced chiropractors evaluated the dose response for chiropractic care of cervicogenic headache [27]. The participants were diagnosed according to the IHS criteria except the radiographic criterion [1]. Participants were excluded if SMT was contraindicated or if the participants had complicated condition that might have been related to clinical outcome. The primary end-point was a change in mean headache intensity from baseline to 4- and 12-week follow-up recorded on 100 points modified Von Korff pain scale. The headache intensity score is the average of headache intensity today, worst headache intensity within the last 4 weeks and average headache intensity within the last 4 weeks. Headache frequency, disability, and neck pain were secondary end-points. Although the participants were allowed to seek consultations outside the trial, only few used that opportunity. The main results of the RCT were that several consultations seem to be advantageous over few consultations in the treatment of cervicogenic headache (Table 2). At 4- and 12-week follow-up headache disability was reduced 44, 50, $76 \%$ and $14,52,55 \%$ in the SMT 1,3 and 4 times a week groups, while neck pain was reduced by $31,50,55 \%$ and $30,54,38 \%$, respectively. Comparison of the SMT 1 time a week group with SMT 3 and 4 times a week groups was not statistically significant. 
Table 2 Criteria list of methodological quality assessment of randomized controlled trials (RCTs) [22]

1. Study population (30 points)

a) Description of inclusion and exclusion criteria (1 point). Restriction to a homogeneous study population (1 point)

b) Comparability of relevant baseline characteristics: duration of complaint (1 point), value of outcome measures (1 point), age (1 point), recurrences (1 point), and radiating complaints/associated symptoms (1 point)

c) Description of the randomization procedure (2 points). Randomization procedure which excluded bias, i.e. random numbers table

(2 points)

d) Description of dropouts for each group and their reasons (3 points)

e) Loss to follow-up: less than $20 \%$ loss to follow-up ( 2 points), OR less than $10 \%$ loss to follow-up (4 points)

f) Sample size: greater than 50 subjects in the smallest group after randomization (6 points), OR greater than 100 subjects in the smallest group after randomization (12 points)

2. Interventions (30 points)

g) Correct description of the manual intervention (5 points). All interventions described (5 points)

h) Pragmatic study: comparison with an existing treatment modality (5 points)

i) Co-interventions avoided in the design of the study (5 points)

j) Comparison with a placebo control group (5 points)

k) Mention of the experience of the therapist (5 points)

3. Measurement of effect (30 points)

1) Placebo controlled studies: patients blinded ( 3 points), blinding evaluated and fully successful (2 points) OR Pragmatic studies: patients fully naive, evaluated and fully successful ( 3 points), time restriction of no manual treatments for at least 1 year ( 2 points)

m) Outcome measures: pain assessment ( 2 points), global measure of improvement ( 2 points), functional status ( 2 points), spinal mobility ( 2 points), medical consumption ( 2 points)

n) Each blinded outcome measure mentioned under item $M$ earns 2 points

o) Analysis of post-treatment data ( 3 points), inclusion of a follow-up period longer than 6 months ( 2 points)

4. Data presentation and analysis (10 points)

p) Intention-to-treat analysis when loss to follow-up is less than $10 \%$ OR intention-to-treat analysis as well as worst-case analysis for missing values when loss to follow-up is greater than $10 \%$ (5 points)

q) Corrected presentation of the data: mean or median with a standard deviation or percentiles for continuous variables (5 points)

Table 3 Quality score of the analyzed randomized controlled trials (RCTs) using manual therapies for treatment of CEH

\begin{tabular}{llllllllllllllllllll}
\hline Study & A & B & C & D & E & F & G & H & I & J & K & L & M & N & O & P & Q & Total \\
\hline Piekartz and Lüdtke [30] & 2 & 3 & 4 & 3 & 2 & 0 & 10 & 5 & 0 & 0 & 5 & 2 & 6 & 6 & 3 & 0 & 5 & 56 \\
Nilsson [24] & 2 & 2 & 4 & 3 & 4 & 0 & 10 & 5 & 5 & 0 & 0 & 2 & 4 & 4 & 3 & 0 & 5 & 53 \\
Nilsson et al. [25] & 2 & 2 & 4 & 3 & 4 & 0 & 10 & 5 & 5 & 0 & 0 & 2 & 4 & 4 & 3 & 0 & 5 & 53 \\
Jull et al. [26] & 2 & 5 & 4 & 3 & 4 & 0 & 10 & 5 & 5 & 5 & 5 & 2 & 8 & 8 & 5 & 5 & 5 & 81 \\
Haas et al. [27] & 2 & 4 & 4 & 3 & 4 & 0 & 10 & 5 & 0 & 0 & 5 & 2 & 6 & 0 & 3 & 5 & 5 & 58 \\
Borusiak et al. [28] & 2 & 2 & 4 & 0 & 4 & 0 & 10 & 0 & 5 & 5 & 5 & 2 & 6 & 0 & 0 & 0 & 5 & 50 \\
Haas et al. [29] & 2 & 4 & 4 & 3 & 4 & 0 & 10 & 5 & 0 & 5 & 5 & 2 & 6 & 0 & 3 & 5 & 5 & 63 \\
\hline
\end{tabular}

The letters correspond with letters from the criteria list (Table 2)

The German study was conducted by a physician with blinded participants and unblended treatment and outcome measures [28]. The study followed the guidelines of the IHS with slight modifications, as the diagnosis $\mathrm{CEH}$ according to the criteria can only be given retrospectively after resolution of the symptoms [5]. Participants were allowed to have co-occurrence of migraine and/or tensiontype headache. Participants were excluded if ever exposed to SMT or diagnosed with secondary headaches other than
CEH. Main outcome measures were headache frequency, duration, intensity, medication consumption and days of absence from school. No statistical significant change was observed in the treatment or sham group between baseline and at 2-month follow-up in relation to medicine consumption or days of absence from school due to headache.

The 2nd American pilot study was conducted by four experienced chiropractors while additional chiropractors in 


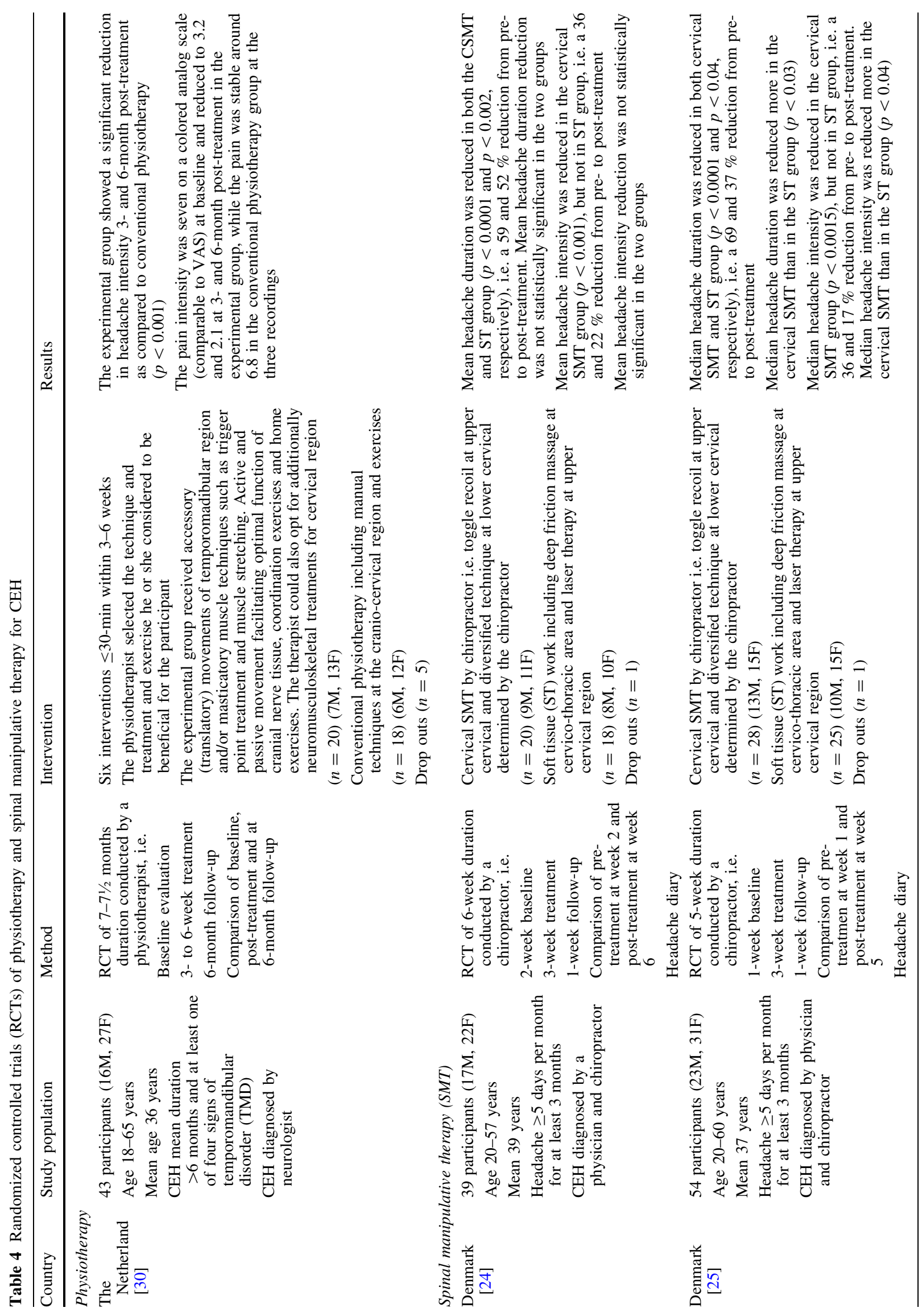




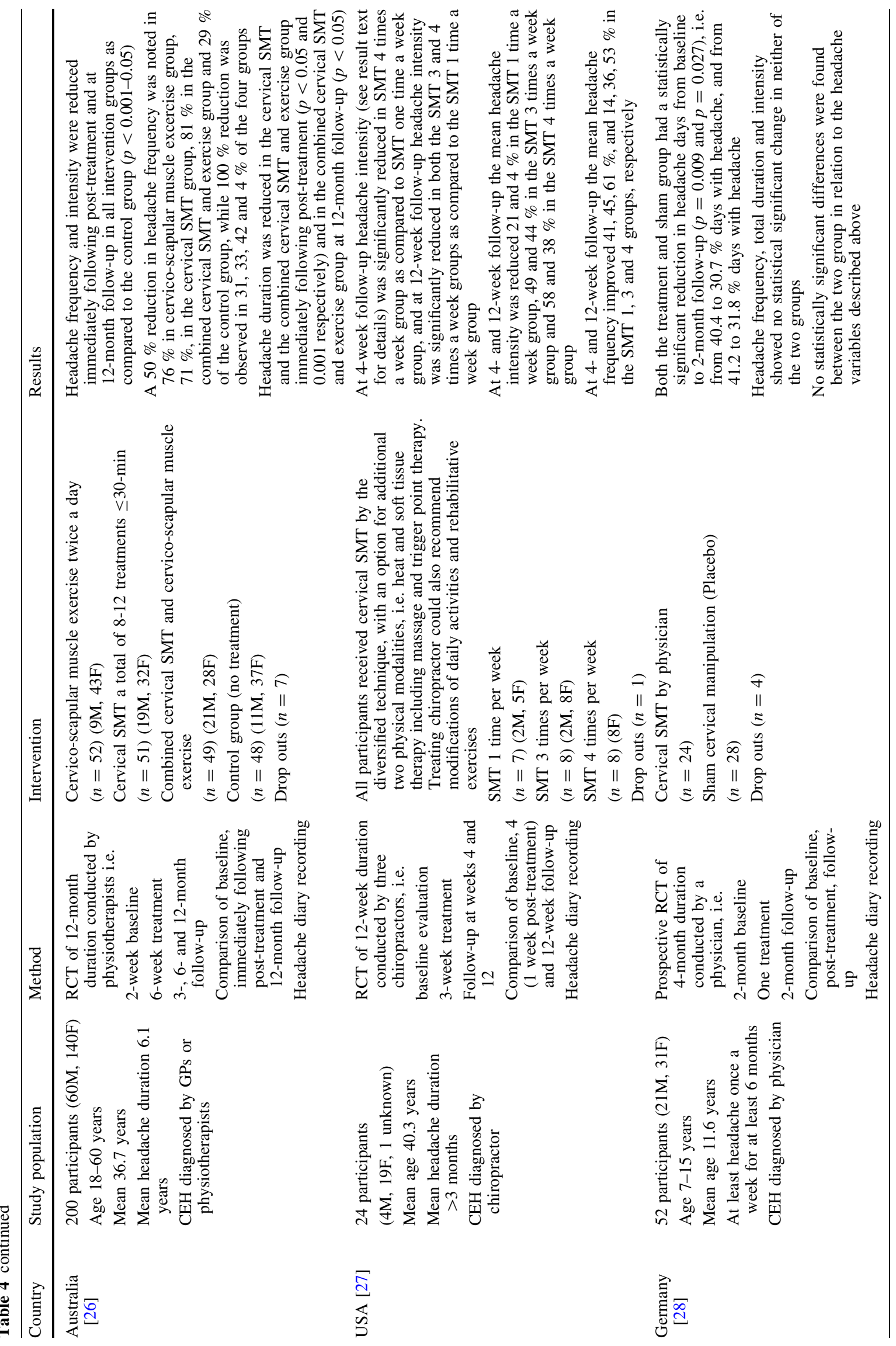




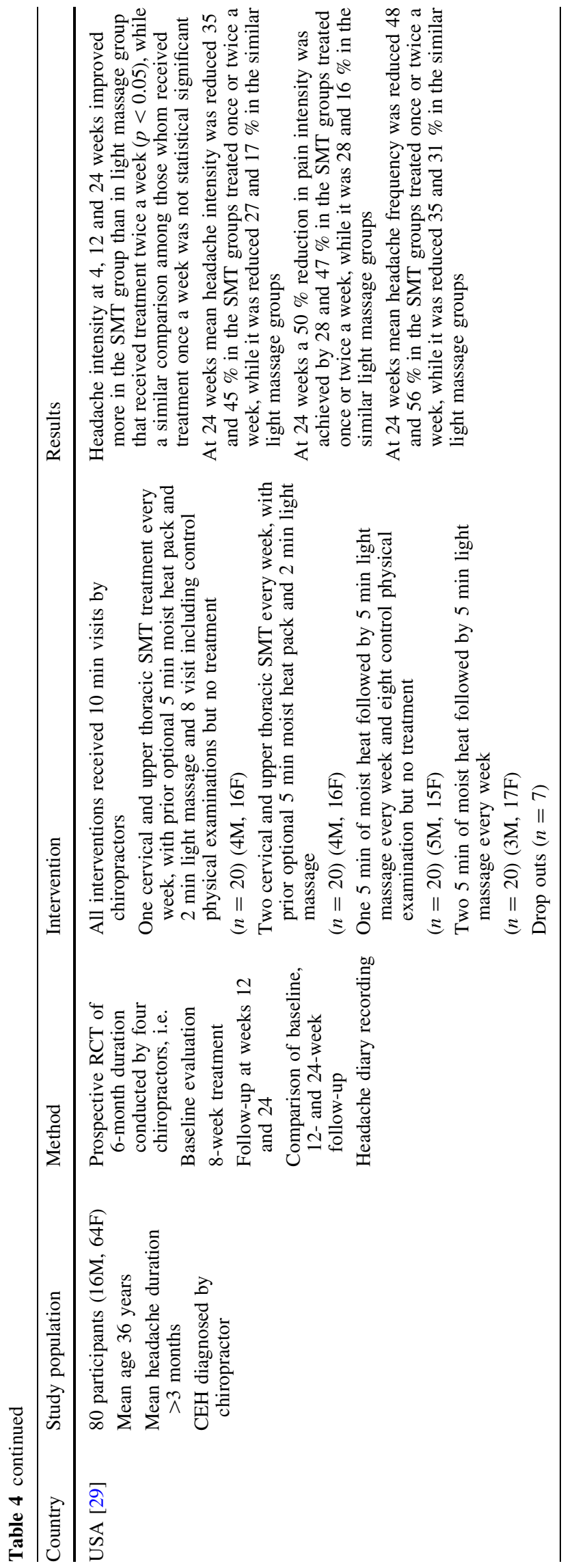

each clinic served as a backup therapist [29]. The treatment and outcome measures were unblinded. Participants were diagnosed according to the IHS excluding the radiographic criteria using a questionnaire [5]. Participants were excluded if they could not attend two visits per week for 8 weeks, took prophylactic prescribed medication for headache, had massage or SMT for their headache within the last 3 months or had complicated conditions. The primary end-point was headache intensity while secondary end-points were headache frequency, disability, neck pain and use of over the counter medication (OTC). At 24 weeks mean neck pain and mean neck disability were reduced 28 and $52 \%$ in the SMT group treated once a week, 47 and $52 \%$ in the SMT group treated twice a week, 29 and $45 \%$ in the light massage (LM) group treated once a week, and 18 and $20 \%$ in the LM group treated twice a week. The authors concluded that only the SMT group treated twice a week had clinical important effect on mean neck pain and disability. Generally dose effects tended to be small.

\section{Discussion}

\section{Methodological considerations}

All seven RCTs studies ascertained the participants through clinical interviews which is considered to be the most valid method in establishing a precise headache diagnosis [31]. All the RCTs included relatively few participants except the Australian physiotherapy study [26]. However, due to participants were divided into four groups each with 48-52 participants, even the Australian study did not receive points for number of participants in the quality score (Table 3). The number of investigators in the seven RCTs varied from 1 to 25 . The advantage with one investigator is elimination of inter-observer variability, which is likely to be present if there are two or more investigators. The 25 investigators in the Australian study might be a challenge in relation to the result [32]. The Dutch study was flawed by the participants not being blinded to the intervention, as well as co-intervention was allowed by the investigator which is a major risk for bias [30]. All the RCTs were considered to be of at least good methodological quality, i.e. score $\geq 50$ (Table 3 ), with the Australian study standing out with an excellent 81 points score of the maximum 100 points.

According to the guidelines of the IHS, an intervention is recommended to last at least 3 months in chronic migraine trials [33]. All the RCTs had less than 3-month intervention, varying from a single treatment to 8 -week treatment. In three of SMT the RCTs allowed non-trial treatment which can lead to biased results [26, 27, 29]. Two of the RCTs included participants with co-occurrence 
of migraine and tension-type headache [28, 29], thus, the effect observed might not be exclusively due to improvement of the $\mathrm{CEH}$.

Only one of the RCTs included a control group that did not receive treatment [26]. It is generally accepted that RCTs including a control group are advantages to pragmatic RCTs, as the effect in the placebo control group often is high [23]. True net effect is more accurately calculated when adding a control group. One RCT had had a successful blinding using SMT or sham treatment, the latter group was denoted as "control group" by the authors [28]. Future RCTs should include a placebo group, i.e. a group of participant that do not receive treatment, although, it is known that blinding adult participants in SMT trials is difficult [34]. Thus, the lack of control group that do not receive treatment makes interpretation of the results difficult, since many of the RCTs had "control groups" that receive a non SMT treatment that might had some effect.

\section{Results}

The Dutch study was considered to be of good methodological quality, although it had room for many improvements [30]. The experimental group had a statistically significant improvement in headache intensity as compared to conventional physiotherapy, an effect that must be considered to also be of clinical significance as the headache intensity was reduced $>50 \%$. The study stands alone, since it also included TMD complaints that also improved. The study included multimodal treatment modalities such as exercise, and thus the results cannot with certainty be exclusive of manual intervention.

The two Danish studies were based on the same study population, with additional 15 participants in the 2nd Danish study [24, 25]. The 1st Danish study presented mean data and the 2nd Danish study presented median data. The median but not mean headache duration and intensity was statistically significantly reduced in the SMT group as compared to the ST group [24, 25]. The 59 and $52 \%$ mean reduction of headache duration in the SMT and ST groups is clinically meaningful, and the 36 and $22 \%$ mean reduction in headache intensity in the two groups is also likely to be clinically meaningful.

The Australian study showed a significant reduction in headache frequency and intensity in all active treatment groups as compared to the control group, an effect that was maintained at 12-month follow-up [26].

The 1st American RCT was a dose-response study without statistical significant results, but there was a tendency toward favouring SMT three or four times a week for SMT once a week [27]. The study did not avoid cointervention in the any of the three groups leading to a possible bias.
The German RCT included children and adolescent and had only one treatment session, and found no statistically significant differences [28]. Due to the single treatment, it cannot be excluded that more treatment sessions might have given another result, considering that $\mathrm{CEH}$ is known to be difficult to treat.

The results of the 2nd American study favoured SMT for light massage (LM), and favouring SMT four times a week slightly over SMT three times a week [29].

One of the major problems in all the RCTs is the fact that the majority of participants had intermittent $\mathrm{CEH}$ [24-30]. However, CEH is often characterized by a continuous headache with an intensity that might fluctuate rather than being a paroxysmal disorder [10, 14]. The fact that $\mathrm{CEH}$ is often continuous makes sense, assuming that $\mathrm{CEH}$ is caused by local factors in the neck/cervical spine. Another major problem is the fact that clinical diagnostic criteria for $\mathrm{CEH}$ have not proved to be valid [35]. Although, applying the diagnosis criteria of CHISG not including a blockage of the greater occipital nerve (GON) is equally inter-observer reliable as the diagnosis of migraine and tension-type headache [36]. Thus, the validity of a GON blockage as a diagnostic criteria can be questioned. Medication is usually ineffective in CEH. So far there have not been conducted any RCTs on the effect of medicine in CEH. Blockage of the GON might be effective in $\mathrm{CEH}[10,18,19]$. However, an operation of the peripheral course of GON with special attention to the trapezius insertion had no effect [37].

\section{Conclusion}

Current RCTs suggest that physiotherapy and SMT might be an effective treatment in the management of $\mathrm{CEH}$. However, the RCTs mostly included participant with infrequent $\mathrm{CEH}$. Future challenges regarding $\mathrm{CEH}$ are substantial both from a diagnostic and management point of view.

\section{Conflict of interest None.}

Open Access This article is distributed under the terms of the Creative Commons Attribution License which permits any use, distribution, and reproduction in any medium, provided the original author(s) and the source are credited.

\section{References}

1. Headache Classification Committee of the International Headache Society (1988) Classification and diagnostic criteria for headache disorders, cranial neuralgias and facial pain. Headache Classification Committee of the International Headache Society. Cephalalgia 8(Suppl 7):1-96 
2. Sjaastad O, Fredriksen TA, Pfaffenrath V (1990) Cervicogenic headache: diagnostic criteria. Headache 30(11):725-726

3. Sjaastad O, Fredriksen TA, Pfaffenrath V (1998) Cervicogenic headache: diagnostic criteria. The Cervicogenic Headache International Study Group. Headache 38(6):442-445

4. Sjaastad O, Fredriksen TA (2000) Cervicogenic headache: criteria, classification and epidemiology. Clin Exp Rheumatol 18(2 Suppl 19):S3-S6

5. Headache Classification Subcommittee of the International Society (2004) The international classification of headache disorders: 2nd edition. Cephalalgia 24(Suppl 1):9-160

6. Nilsson N (1995) The prevalence of cervicogenic headache in a random population sample of 20-59 year olds. Spine (Phila Pa 1976) 20(17):1884-1888

7. Pareira Monteriro J. Epidemiologic e clinico de uma populacäo urbana University of Porto, Cefaleia; 1995

8. Sjaastad O, Bakketeig LS (2008) Prevalence of cervicogenic headache: Vaga study of headache epidemiology. Acta Neurol Scand 117(3):173-180

9. Aaseth K, Grande RB, Kvaerner KJ, Gulbrandsen P, Lundqvist C, Russell MB (2008) Prevalence of secondary chronic headaches in a population-based sample of 30-44-year-old persons. The Akershus study of chronic headache. Cephalalgia 28(7):705-713

10. Knackstedt H, Bansevicius D, Aaseth K, Grande RB, Lundqvist C, Russell MB (2010) Cervicogenic headache in the general population: the Akershus study of chronic headache. Cephalalgia 30(12):1468-1476

11. Kerr FW (1972) Central relationships of trigeminal and cervical primary afferents in the spinal cord and medulla. Brain Res 43(2):561-572

12. Bogduk N (2004) The neck and headaches. Neurol Clin 22(1): 151-71, vii

13. Bogduk N (1992) The anatomical basis for cervicogenic headache. J Manipulative Physiol Ther 15(1):67-70

14. Knackstedt H, Krakenes J, Bansevicius D, Russell MB (2012) Magnetic resonance imaging of craniovertebral structures: clinical significance in cervicogenic headaches. J Headache Pain 13(1):39-44

15. Diener HC, Kaminski M, Stappert G, Stolke D, Schoch B (2007) Lower cervical disc prolapse may cause cervicogenic headache: prospective study in patients undergoing surgery. Cephalalgia 27(9):1050-1054

16. Grande RB, Aaseth K, Benth JS, Lundqvist C, Russell MB (2011) Reduction in medication-overuse headache after short information. The Akershus study of chronic headache. Eur J Neurol 18(1):129-137

17. Aaseth K, Grande RB, Benth JS, Lundqvist C, Russell MB (2011) 3-Year follow-up of secondary chronic headaches: The Akershus study of chronic headache. Eur J Pain 15(2):186-192

18. Vincent MB, Luna RA, Scandiuzzi D, Novis SA (1998) Greater occipital nerve blockade in cervicogenic headache. Arq Neuropsiquiatr 56(4):720-725

19. Inan N, Ceyhan A, Inan L, Kavaklioglu O, Alptekin A, Unal N (2001) C2/C3 nerve blocks and greater occipital nerve block in cervicogenic headache treatment. Funct Neurol 16(3):239-243

20. Ter Riet G, Kleijnen J, Knipschild P (1990) Acupuncture and chronic pain: a criteria-based meta-analysis. J Clin Epidemiol 43(11):1191-1199

21. Koes BW, Assendelft WJ, van der Heijden GJ, Bouter LM, Knipschild PG (1991) Spinal manipulation and mobilisation for back and neck pain: a blinded review. BMJ 303(6813):12981303

22. Fernandez-de-las-Penas C, Alonso-Blanco C, San-Roman J, Miangolarra-Page JC (2006) Methodological quality of randomized controlled trials of spinal manipulation and mobilization in tension-type headache, migraine, and cervicogenic headache. J Orthop Sports Phys Ther 36(3):160-169

23. Chaibi A, Tuchin PJ, Russell MB (2011) Manual therapies for migraine: a systematic review. J Headache Pain 12(2):127-133

24. Nilsson N (1995) A randomized controlled trial of the effect of spinal manipulation in the treatment of cervicogenic headache. J Manipulative Physiol Ther 18(7):435-440

25. Nilsson N, Christensen HW, Hartvigsen J (1997) The effect of spinal manipulation in the treatment of cervicogenic headache. J Manipulative Physiol Ther 20(5):326-330

26. Jull G, Trott P, Potter H, Zito G, Niere K, Shirley D et al (2002) A randomized controlled trial of exercise and manipulative therapy for cervicogenic headache. Spine (Phila $\mathrm{Pa}$ 1976) 27(17): 1835-1843

27. Haas M, Groupp E, Aickin M, Fairweather A, Ganger B, Attwood $M$ et al (2004) Dose response for chiropractic care of chronic cervicogenic headache and associated neck pain: a randomized pilot study. J Manipulative Physiol Ther 27(9):547-553

28. Borusiak P, Biedermann H, Bosserhoff S, Opp J (2010) Lack of efficacy of manual therapy in children and adolescents with suspected cervicogenic headache: results of a prospective, randomized, placebo-controlled, and blinded trial. Headache 50(2):224-230

29. Haas M, Spegman A, Peterson D, Aickin M, Vavrek D (2010) Dose response and efficacy of spinal manipulation for chronic cervicogenic headache: a pilot randomized controlled trial. Spine J 10(2):117-128

30. Von Piekartz H, Ludtke K (2011) Effect of treatment of temporomandibular disorders (TMD) in patients with cervicogenic headache: a single-blind, randomized controlled study. Cranio 29(1):43-56

31. Rasmussen BK, Jensen R, Olesen J (1991) Questionnaire versus clinical interview in the diagnosis of headache. Headache 31(5):290-295

32. Harris-Hayes M, Van Dillen LR (2009) The inter-tester reliability of physical therapists classifying low back pain problems based on the movement system impairment classification system. PM R 1(2):117-126

33. Silberstein S, Tfelt-Hansen P, Dodick DW, Limmroth V, Lipton RB, Pascual J et al (2008) Task Force of the International Headache Society Clinical Trial Subcommittee. Guidelines for controlled trials of prophylactic treatment of chronic migraine in adults. Cephalalgia 28(5):484-495

34. Vernon H, MacAdam K, Marshall V, Pion M, Sadowska M (2005) Validation of a sham manipulative procedure for the cervical spine for use in clinical trials. J Manipulative Physiol Ther 28(9):662-666

35. Bogduk N, Govind J (2009) Cervicogenic headache: an assessment of the evidence on clinical diagnosis, invasive tests, and treatment. Lancet Neurol 8(10):959-968

36. van Suijlekom JA, de Vet HC, van den Berg SG, Weber WE (1999) Interobserver reliability of diagnostic criteria for cervicogenic headache. Cephalalgia 19(9):817-823

37. Bovim G, Fredriksen TA, Stolt-Nielsen A, Sjaastad O (1992) Neurolysis of the greater occipital nerve in cervicogenic headache. A follow up study. Headache 32(4):175-179 